\title{
Factores asociados a la disponibilidad y uso de medios electrónicos en niños desde preescolar hasta $4^{\circ}$ grado
}

\author{
Diana Marina Camargo ${ }^{1}$, Luis Carlos Orozco² \\ 1 Escuela de Fisioterapia, Universidad Industrial de Santander, Bucaramanga, Colombia \\ 2 Escuela de Enfermería, Universidad Industrial de Santander, Bucaramanga, Colombia \\ Introducción. El incremento en la disponibilidad de medios electrónicos ha modificado los \\ comportamientos de niños y jóvenes, fomentando los de tipo sedentario, con efectos sobre la salud \\ desde muy corta edad. \\ Objetivo. Caracterizar la disponibilidad y uso de medios electrónicos, y sus factores asociados, en \\ niños desde preescolar hasta $4^{\circ}$ grado. \\ Materiales y métodos. Es un estudio de corte transversal con muestreo por conglomerados. Los \\ padres diligenciaron la encuesta sociodemográfica, disponibilidad y uso de medios electrónicos en \\ el hogar y en la habitación del niño. Se aplicaron modelos de regresión log-binomial para estimar las \\ razones de prevalencia, ajustando por el tipo de muestreo. \\ Resultados. Respondieron 710 padres. El promedio de edad de los niños fue de 6,7 años y 49,7\% eran \\ hombres. El trabajo fuera de la casa de la mamá y los ingresos familiares se asociaron positivamente \\ con la disponibilidad de medios electrónicos en el hogar y en la habitación del hijo. La disponibilidad de \\ televisor, computador y consolas en la habitación del hijo, favorece un mayor tiempo de uso de estos \\ medios electrónicos. Tanto el sexo masculino del hijo como la edad, se asociaron positivamente con la \\ disponibilidad y uso de los medios electrónicos. \\ Conclusión. Este es el primer estudio en Colombia en que se documentan la disponibilidad y el uso \\ de medios electrónicos en niños. Los hallazgos reflejan que la vida moderna fomenta comportamientos \\ sedentarios desde los primeros años de vida, asociados con problemas de salud desde la primera \\ infancia. Se requieren estudios de intervención dirigidos a disminuir estos comportamientos en nuestro \\ contexto.
}

Palabras clave: niño, preescolar, tecnología, televisión, estilo de vida sedentario, conducta infantil, bienestar del niño.

doi: http://dx.doi.org/10.7705/biomedica.v33i2.779

\section{Associated factors to availability and use of electronic media in children from preschool to $4^{\text {th }}$ grade}

Introduction: The increased availability of electronic media has changed the behavior in children and young people by encouraging sedentary lifestile, with health effects from a very young age.

Objective: To characterize the availability and use of electronic media and its associated factors in children from preschool through $4^{\text {th }}$ grade.

Materials and methods: A cross-sectional study with cluster sampling was carried out. Parents filled out the demographic survey, the availability and use of electronic media at home and in the child's room. Log-binomial regression models were applied to estimate prevalence ratios adjusted for the sampling.

Results: Seven hundred and ten parents answered. The average age of the children was 6.7 years and $49.7 \%$ were male. Factors such as the mother working out of the house and family income were positively associated with the availability of electronic media at home and in the child's room. The availability of a TV, computer and console in the child's room, contributes to longer use of these electronic media. Both, the male gender and age of the child, were positively associated with the availability and use of electronic media.

Conclusion: This is the first study in Colombia that reports the availability and use of electronic media in children. It is clear that modern life encourages sedentary behavior from the earliest years of life, which has been associated with health problems from early childhood. Intervention studies are needed aimed at reducing these behaviors in our context.

Contribución de los autores:

Ambos autores participaron en el diseño del proyecto de investigación, análisis de la información y elaboración del artículo. Diana Marina Camargo coordinó el trabajo de campo. 
Key words: Child; child, preschool; technology, television, sedentary lifestyle, child behavior, child welfare.

doi: http://dx.doi.org/10.7705/biomedica.v33i2.779

Los patrones de comportamiento de los niños desde edades muy tempranas, han sufrido grandes cambios en las últimas décadas, pasando de estilos de vida activos en los que predominaba el juego y el deporte, a comportamientos de tipo sedentario como ver televisión y usar el computador. Este fenómeno posiblemente está influenciado por el desarrollo de nuevas tecnologías, la disponibilidad de la televisión y programas de diferente tipo dirigidos al entretenimiento de los niños desde los primeros años de edad, al aumento de factores como la inseguridad en las grandes urbes y al ingreso de las mujeres al medio laboral, entre otros. Asimismo, los currículos en las instituciones educativas han implementado modificaciones que involucran menos tiempo de dedicación a comportamientos activos como el juego, el deporte y las clases de educación física (1).

Las consecuencias de este fenómeno ya se empiezan a reflejar en indicadores de morbilidad, como el sobrepeso y la obesidad en los niños desde los tres años de edad. Según la Organización Mundial de la Salud (OMS), en 2010 había 42 millones de niños con sobrepeso en todo el mundo, de los cuales de 35 millones vivían en países en desarrollo. Se plantea que la causa fundamental es el desequilibrio entre la ingesta y el gasto calórico, debido al mayor consumo de alimentos hipercalóricos con abundantes grasas y azúcares, y una clara tendencia a la disminución de la actividad física, representada por la naturaleza sedentaria de muchas actividades recreativas, por el cambio en los medios de transporte y por la creciente urbanización (2).

En Colombia en el 2010, según la Encuesta Nacional de la Situación Nutricional (ENSIN) (3), se encontró que la prevalencia de sobrepeso u obesidad aumentó 25,9 \% en el último quinquenio; uno de cada seis niños y adolescentes presentaba alguna de estas dos condiciones. Además de los malos hábitos nutricionales, la disminución en los niveles de actividad física en la población,

\footnotetext{
Correspondencia:

Diana Marina Camargo, Escuela de Fisioterapia, Facultad de Salud, Universidad Industrial de Santander, Carrera 32 NN$^{\circ} 29-$ 31, Bucaramanga, Colombia

Teléfono: (57) 634 4000, extensión 3147 y 3381; fax: (57) 635 8582

dcamargo@uis.edu.co
}

Recibido: 16/07/12; aceptado:27/10/12 el incremento de los diversos comportamientos sedentarios, como ver televisión y usar computadores y dispositivos electrónicos, y el tiempo invertido en ellos, contribuyen en forma definitiva al desequilibrio energético que aumenta la probabilidad de presentar sobrepeso y obesidad desde los primeros años de vida (4).

Este es un problema social, pues al contrario de la mayoría de los adultos, los niños y adolescentes no pueden elegir el entorno en el que viven ni los alimentos que consumen. Asimismo, tienen una capacidad limitada para comprender las consecuencias a largo plazo de su comportamiento.

Un estudio en Norteamérica, dirigido a describir el acceso al uso de los medios por niños entre los 0 y los 6 años de edad, mostró que, en un día típico, el $75 \%$ ve televisión y el $32 \%$ videos, por aproximadamente un promedio de 1 hora y 20 minutos diarios; además, el $27 \%$ de los niños entre los 5 y 6 años usan el computador 50 minutos diarios. En relación con la disponibilidad de la televisión, se encontró que una quinta parte de los niños de 0 a 2 y un tercio de los de 5 a 6 años, tienen televisor en su habitación. La mayoría de los niños de 3 a 6 años cumplen con la recomendación de no ver televisión más dos horas diarias, pero el $70 \%$ de aquellos entre 0 y 2 años no la cumplen. Este fenómeno de la población es de vital importancia en la actualidad, porque desde edades muy tempranas los niños se ven expuestos a un ambiente saturado de medios electrónicos, que hacen parte esencial y normativa de su vida diaria y aún no se conoce el impacto que esto pueda tener sobre su vida futura (4).

Desde el punto de vista social, en la actualidad, los niños tienden a preferir el uso de redes sociales por vía internet y dispositivos electrónicos que disminuyen la posibilidad, no solo de mantener un estilo de vida activo, sino de tener interacción social con otros niños de su edad, compartir con sus padres y adaptarse mejor al medio en el que crecen (1).

Colombia no cuenta con estudios que documenten los comportamientos sedentarios en niños de esta franja de edad; por otra parte, se desconoce la magnitud, el tipo y el uso de los diferentes dispositivos y medios electrónicos. Este conocimiento es importante pues contribuirá a entender la dinámica actual de los niños desde edades 
tempranas y plantear posibles intervenciones en forma precoz, no solo para disminuir las cifras de morbilidad ya mencionadas, sino para favorecer comportamientos que se consoliden en estilos de vida saludables y una mejor calidad de vida de la población infantil.

Por lo anterior, el objetivo de este estudio fue caracterizar la disponibilidad y el uso de medios electrónicos en niños desde la etapa preescolar hasta el $4^{\circ}$ grado, en un área urbana de Colombia.

\section{Materiales y métodos}

Se desarrolló un estudio de corte transversal, cuya población estuvo conformada por niños entre preescolar y $4^{\circ}$ grado, matriculados en instituciones educativas públicas y privadas del área urbana de Bucaramanga, Girón y Piedecuesta, así como por sus padres, quienes se encargaron de diligenciar la encuesta. El estudio se desarrolló entre marzo y noviembre de 2011.

\section{Muestreo}

Se aplicó un muestreo por conglomerados, siguiendo la metodología de la OMS para evaluar la cobertura de vacunación (5), seleccionando aleatoriamente 30 instituciones educativas registradas en las secretarías de educación de los municipios ya mencionados, que incluyeran niños desde preescolar hasta grado $4^{\circ}$. Posteriormente, se seleccionaron aleatoriamente entre 30 y 40 estudiantes de cada institución, a quienes se les anexó en la agenda escolar la encuesta dirigida a sus padres, para recolectar la información durante un período máximo de dos días. Por lo anterior, se esperaba contar con una muestra entre 900 y 1.200 niños del rango de edad mencionado.

\section{Criterios de exclusión}

Se excluyeron instituciones con un número de escolares inferior a 100, con el fin de posibilitar la selección aleatoria de los estudiantes previstos, es decir, entre 30 y 40; también, se excluyeron niños con alteraciones físicas o cognitivas de carácter permanente, como síndrome de Down y parálisis cerebral, que por su condición requirieran cuidados especiales y realizaran actividades diferentes a las consideradas normales según el desarrollo motor y cognitivo para su edad.

\section{Variables de estudio}

Variables sociodemográficas de padres e hijos. Se incluyeron sexo (masculino/femenino) y edad (años cumplidos). Además, los padres registraron su escolaridad (años), si trabajaban fuera de la casa o no y qué tipo de dedicación tenía su trabajo (tiempo completo, medio tiempo o inferior a medio tiempo), Se incluyó también una categorización de ingresos mensuales para el núcleo familiar, representado por entradas económicas del padre, la madre, los abuelos, etc. que convivían en la vivienda, en salarios mínimos legales vigentes en cinco categorías $(<1,1-2,3-4,5-6$ y $>6)$. Por otra parte, los padres registraron el grado escolar de su hijo, si vivía con ambos padres o no y si tenía hermanos o no y cuántos.

Información del hogar y de la habitación del niño. Se registró mediante una lista la disponibilidad de medios electrónicos y su cantidad en el hogar (televisión, televisión por cable, computador, DVD, Blu-ray, internet, y consolas de videojuegos como XBox, PlayStation y Wii). Además, se consignó si en su habitación el niño tenía acceso a los mismos equipos, al igual que otros dispositivos electrónicos como celulares y MP3/MP4, entre otros.

Para el análisis del número de medios disponibles, estos se sumaron con base en una respuesta afirmativa paracualquieradelalistay, posteriormente, se calculó la mediana, que permitió generar dos nuevas variables dicotómicas para el hogar y para la habitación del niño.

Uso de medios y tecnología. Se indagó a los padres si estaban de acuerdo o no con cinco afirmaciones diferentes, relacionadas con el uso y control de la televisión en el hogar (6). Además, el padre o acudiente respondió sobre el uso (sí/ no) de diferentes medios por parte de su hijo y, si la respuesta era positiva, se registró el tiempo de dedicación en horas por día. Esta información se consignó por separado para un día típico entre semana y uno de fin de semana.

Para el análisis del tiempo de uso, se acogieron las recomendaciones internacionales sobre actividad física y comportamientos sedentarios emitidas por la American Academy of Pediatrics (7), la National Association for Sport and Physical Education (NASPE) (8) y el Department of Health and Aging (DoHA) del gobierno australiano (9); por lo tanto, se establecieron como puntos de corte, para ver televisión, más de dos horas al día, y para el uso de computador y videojuegos, más de una hora por día.

\section{Procedimiento}

En un trabajo previo se evaluó la reproducibilidad de las preguntas dirigidas a los padres, relacionadas 
con el hábito de la siesta y el uso de diferentes medios electrónicos por parte de sus hijos. Los valores kappa encontrados entre semana y durante el fin de semana, respectivamente, fueron: de $0,56 \mathrm{y}$ 0,36 para ver televisión, de 0,56 y 0,57 para uso de computador, de 0,57 y 0,58 para uso de videojuegos, y de 0,57 y 0,59 para tomar siesta.

Inicialmente se aplicó una prueba piloto en una institución educativa con 30 escolares y sus padres, de características sociodemográficas similares a las establecidas para el trabajo, con el fin de evaluar la claridad de las preguntas del instrumento y aplicar los ajustes pertinentes.

Posteriormente y en forma aleatoria, se seleccionaron mediante un muestreo por conglomerados las instituciones y los correspondientes escolares participantes. Una vez firmada la carta de consentimiento informado por parte de los rectores, se remitieron las encuestas en la agenda de cada estudiante, dirigidas a los padres o acudientes, acompañadas de las cartas de información general del trabajo y del consentimiento informado. Se estableció un intervalo de dos días parala recolección de la información en la institución educativa. Una vez recolectada toda la información, se elaboró la base de datos para su análisis posterior.

\section{Análisis}

La descripción inicial de las variables y del análisis bivariado, incluyó la aplicación de medidas de tendencia central y dispersión, tablas de frecuencia y pruebas estadísticas, según la naturaleza y distribución de cada variable $(10,11)$.

Para el análisis multivariado se establecieron como variables explicativas las características de los padres y del hogar, así como las características sociodemográficas de los niños.

Se definieron cuatro variables de salida en forma dicotómica, de la siguiente forma: 1) disponibilidad de medios electrónicos en el hogar (mediana $\geq 5$ ), 2) disponibilidad de medios electrónicos en el cuarto del niño (mediana $\geq 1$ ), 3) uso de televisor más de dos horas diarias, y 4) uso de consolas/videojuegos y computador por más de una hora al día.

Para explorar posibles asociaciones, se aplicaron modelos de regresión log-binomial simples y múltiples, con el fin de estimar las razones de prevalencia y sus intervalos de confianza del $95 \%$ $\left(\mathrm{IC}_{95 \%}\right)(12,13)$, evaluando la posible colinealidad entre las variables explicativas y siguiendo las recomendaciones de Greenland (14) para la inclusión de las variables en el modelo. Todos los parámetros estimados se ajustaron por el tipo de muestreo. La base de datos se elaboró en Exce ${ }^{\circledR}$ por duplicado para su posterior validación y análisis en Stata $12.0^{\circledR}$, considerando un nivel de significancia a de 0,05 (15).

\section{Consideraciones éticas}

Para el desarrollo del trabajo se solicitó el consentimiento informado por escrito al rector 0 representante legal de la institución educativa, y a los padres o acudientes del menor, previa remisión de la información completa relacionada con el trabajo que se estaba realizando. El proyecto fue aprobado por el Comité de Investigación Científica de la Facultad de Salud de la Universidad Industrial de Santander.

\section{Resultados \\ Descripción inicial}

Participaron 29 instituciones educativas, una de ellas con doble muestra debido al tamaño de la población escolar, para un total de 710 escolares de Bucaramanga, Piedecuesta y Girón, lo que representa un porcentaje de respuesta de $59,2 \%$, dado que se repartieron 1.200 encuestas. Los cuestionarios fueron respondidos primordialmente por la madre $(61,6 \%)$, por ambos padres $(27,6 \%)$, por el padre $(7,5 \%)$ y el resto, por otra persona.

\section{Información de los padres}

Las características de los padres de familia se muestran en el cuadro 1. Vale la pena señalar que todas las preguntas no fueron respondidas en las 710 encuestas aplicadas, por lo cual se anotan el número y el porcentaje respectivo. La variable relacionada con los ingresos mensuales fue respondida por 679 personas, base para el cálculo de las prevalencias e intervalos de confianza del $95 \%$; cabe destacar las mayores frecuencias para los ingresos entre 1 y 4 salarios mínimos legales vigentes.

\section{Información de los niños}

Las características de los niños participantes mostraron una distribución homogénea por sexo para edad y grado escolar (cuadro 2). En cuanto a las características del núcleo familiar, se registró que $563(80,5 \%)$ de los niños vivían con los dos padres y $425(60,8 \%)$ tenían entre uno y siete hermanos, número que osciló con las siguientes frecuencias: un hermano, $303(73,2 \%)$, dos 
Cuadro 1. Características sociodemográficas de los padres que respondieron el cuestionario

\begin{tabular}{|c|c|c|c|}
\hline Variable & $\mathrm{n}^{\star}$ & Madre & Padre \\
\hline Edad (años), mediana (rango) & 696 & $33(19-53)$ & $36(20-76)$ \\
\hline Escolaridad (años), mediana (rango) & 699 & $13(1-23)$ & $11(1-20)$ \\
\hline Trabaja fuera de la casa, $\mathrm{n}(\%)$ & 695 & $448 \quad(64,5)$ & $606 \quad(92,8)$ \\
\hline Menos de medio tiempo, $n(\%)$ & & $24 \quad(5,4)$ & $10 \quad(1,7)$ \\
\hline Medio tiempo, n (\%) & & $116(26,2)$ & $(9,4)$ \\
\hline Tiempo completo, n (\%) & & $303(68,4)$ & $537 \quad(88,1)$ \\
\hline $\begin{array}{l}\text { Ingresos económicos familiares mensuales } \\
(\mathrm{SMLV}) \text {, porcentaje }\left(\mathrm{IC}_{95 \%}\right)\end{array}$ & 679 & & \\
\hline Menos de 1 & & \multicolumn{2}{|c|}{$10,5 \quad(6,5-14,4)$} \\
\hline Entre 1 y 2 & & \multicolumn{2}{|c|}{$43,7(34,9-52,5)$} \\
\hline Entre 3 y 4 & & \multicolumn{2}{|c|}{$30,9(24,6-37,3)$} \\
\hline Entre 5 y 6 & & \multicolumn{2}{|c|}{$9,7 \quad(5,5-13,9)$} \\
\hline Más de 6 & & \multicolumn{2}{|c|}{$5,1 \quad(1,5-8,8)$} \\
\hline
\end{tabular}

*n: número de personas que respondieron la pregunta; SMLV: salarios mínimos legales vigentes

Cuadro 2. Características sociodemográficas de los niños participantes por sexo

\begin{tabular}{lccc}
\hline Característica & $\begin{array}{c}\text { Niñas } \\
\mathbf{n = 3 5 7}\end{array}$ & $\begin{array}{c}\text { Niños } \\
\mathbf{n = 3 5 3}\end{array}$ & $\begin{array}{c}\text { Total } \\
\mathbf{N = 7 1 0}\end{array}$ \\
\hline Edad (años) & & & \\
3 & 19 & 23 & 42 \\
4 & 32 & 32 & 64 \\
5 & 47 & 47 & 94 \\
6 & 61 & 54 & 115 \\
7 & 61 & 56 & 117 \\
8 & 61 & 74 & 135 \\
9 & 56 & 43 & 99 \\
$\geq 10$ & 20 & 24 & 44 \\
Grado escolar $\mathrm{N}^{\circ}$ & & & \\
Prejardín & 30 & 30 & 60 \\
Jardín & 32 & 47 & 79 \\
Transición & 44 & 34 & 78 \\
1 & 66 & 57 & 123 \\
2 & 59 & 67 & 126 \\
3 & 65 & 65 & 130 \\
4 & 61 & 3 & 114 \\
\hline
\end{tabular}

hermanos, $89(21,5 \%)$, y tres o más hermanos, $22(5,3 \%)$.

\section{Información del hogar y de la habitación del niño}

La información relacionada con la disponibilidad de recursos electrónicos en el hogar y en la habitación del niño, se muestra en la figura 1 . Se destaca el predominio del televisor, con una mediana de dos por hogar, con un rango entre 1 y 6 ; en relación con los computadores y DVD, se registró para ambos una mediana de uno con el mismo rango encontrado para los televisores; igual mediana se encontró para el número de Blu-ray, con un rango entre 1 y 2. Además, se indagó por la disponibilidad de teléfonos y dispositivos móviles por parte de los

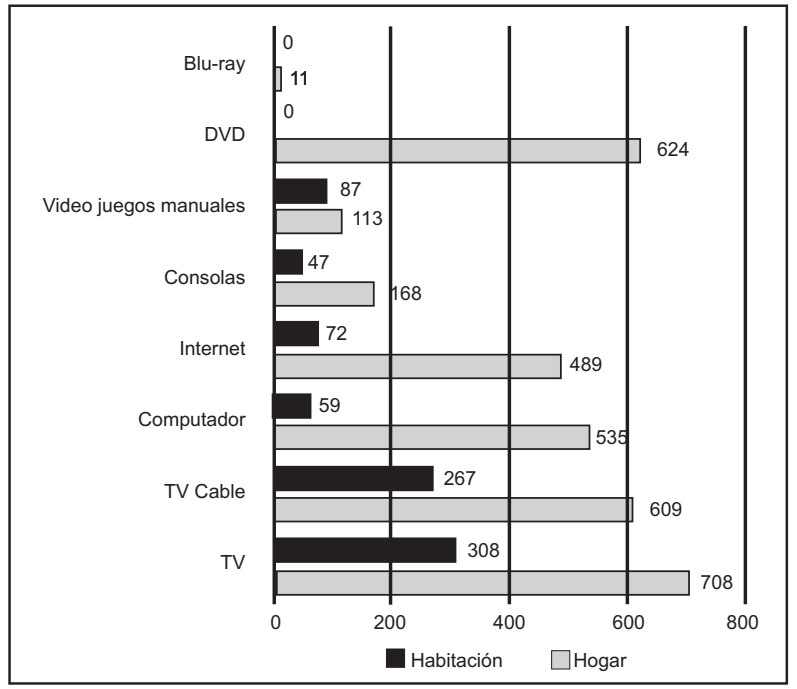

Figura 1. Disponibilidad de medios electrónicos en la habitación del niño y en el hogar

niños, encontrándose que $166(23,8 \%)$ contaban con celular, $3(0,5 \%)$, con blackberry, $57(8,6 \%)$, con reproductores MP3/4, 6 (0,9\%), con Ipod, y 8 (1,3\%), con otros, como parlantes con USB y grabadoras.

\section{Uso de medios electrónicos y normas para televisión por parte de los padres}

El análisis sobre el uso de medios electrónicos entre semana y los fines de semana, respectivamente, registró cifras similares: ver televisión, 96,1\% y $96,6 \%$, computadores, $56,4 \%$ y $58,3 \%$, consolas, $10,7 \%$ y $14,3 \%$, y videojuegos, $15,6 \%$ y $18,4 \%$,.

En cuanto a los resultados sobre los conceptos y normas de los padres sobre la utilización del televisor por parte de los hijos, se encontró que 295 (43,3 \%) pensaban que la televisión ayuda en 
los procesos de aprendizaje, 233 (34,7\%), que la televisión es buena, 687 (97,7\%), que es necesario tener reglas para ver televisión, 538 (78,2 \%), que el límite máximo para ver televisión debe ser de dos horas diarias, y 497 (71,6\%) registró que su hijo solo veía los programas que le parecían adecuados a los padres.

\section{Análisis bivariado}

Disponibilidad de medios electrónicos según el sexo de los niños. El análisis sobre la disponibilidad de medios electrónicos en la habitación de los niños de acuerdo con el sexo, mostró asociaciones positivas y estadísticamente significativas para el sexo masculino en cuanto al uso de consolas y videojuegos manuales, y una asociación marginal para el computador, comparado con el femenino (figura 2).

Disponibilidad de medios electrónicos según la edad de los niños. En relación con la disponibilidad de medios electrónicos por edad, es claro su incremento con la edad de los niños, particularmente para las consolas, videojuegos manuales, celulares y dispositivos de tipo MP3/ MP4, todos estadísticamente significativos. Cabe destacar la frecuencia de celulares entre los tres y cinco años, así como de computador e internet en la habitación para la misma edad (figura 3).

\section{Análisis multivariado}

Factores asociados a la disponibilidad de medios electrónicos en el hogar. Se encontraron como

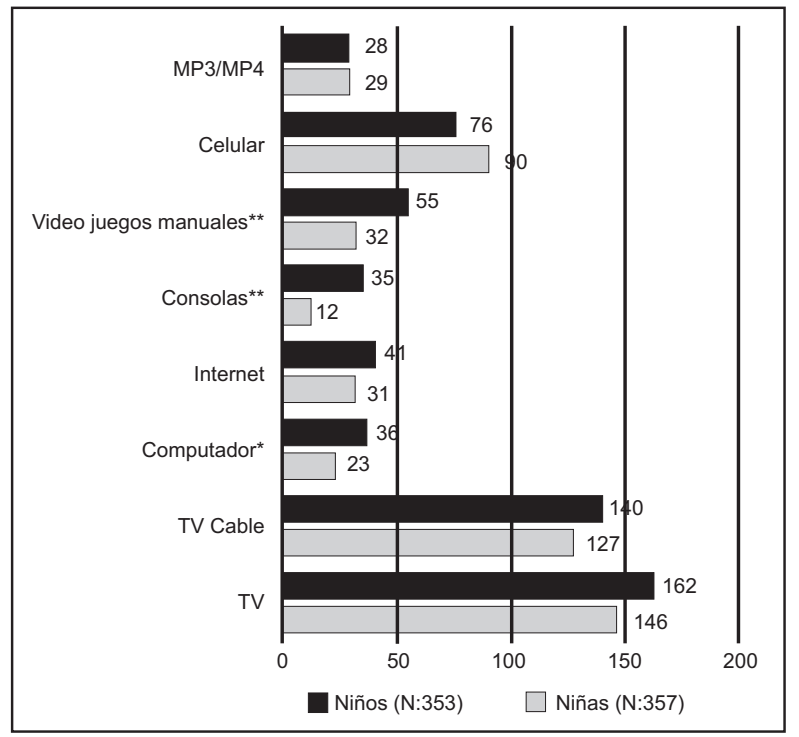

Figura 2. Disponibilidad de medios electrónicos en la habitación de los niños según el sexo $\left({ }^{*} p=0,07,{ }^{* *} p<0,007\right)$. factores asociados positiva y estadísticamente significativos, la escolaridad de la mamá, el nivel de ingresos familiares, el sexo masculino y la edad del hijo (cuadro 3). El modelo mostró un buen ajuste (Linktest 0,52) y contó con 670 observaciones.

Factores asociados a la disponibilidad de medios electrónicos en la habitación del niño. En relación con esta variable, el trabajo de ambos padres fuera de la casa y la edad del hijo, aumentan la probabilidad de disponer de estos dispositivos en la habitación del niño. Por el contrario, contar con hermanos la disminuye (cuadro 4). Este modelo contó con 631 observaciones y con un buen ajuste (Linktest 0,54).

Factores asociados al uso de televisión más de dos horas diarias. Se registró una prevalencia de

Cuadro 3. Factores asociados a la disponibilidad de medios electrónicos en el hogar (variable de salida, mediana $\geq 5$ )

\begin{tabular}{lccr}
\hline Variable & RP & IC 95 \% & \multicolumn{1}{c}{$\mathbf{P}$} \\
\hline $\begin{array}{l}\text { Escolaridad de la } \\
\text { mamá (años) }\end{array}$ & 1,03 & $1,01-1,05$ & $<0,0001$ \\
SMLV & & & \\
$\quad<1$ & 1,0 & & \\
1 & 1,50 & $0,99-2,31$ & 0,06 \\
2 & 1,97 & $1,33-2,90$ & 0,001 \\
$\quad 3-4$ & 2,33 & $1,57-3,47$ & $<0,0001$ \\
Sexo masculino hijo & 1,14 & $1,02-1,26$ & 0,015 \\
Edad hijo (años) & 1,03 & $1,00-1,07$ & 0,028 \\
\hline
\end{tabular}

N=670 observaciones; Linktest: 0,52; SMLV: salarios mínimos legales vigentes

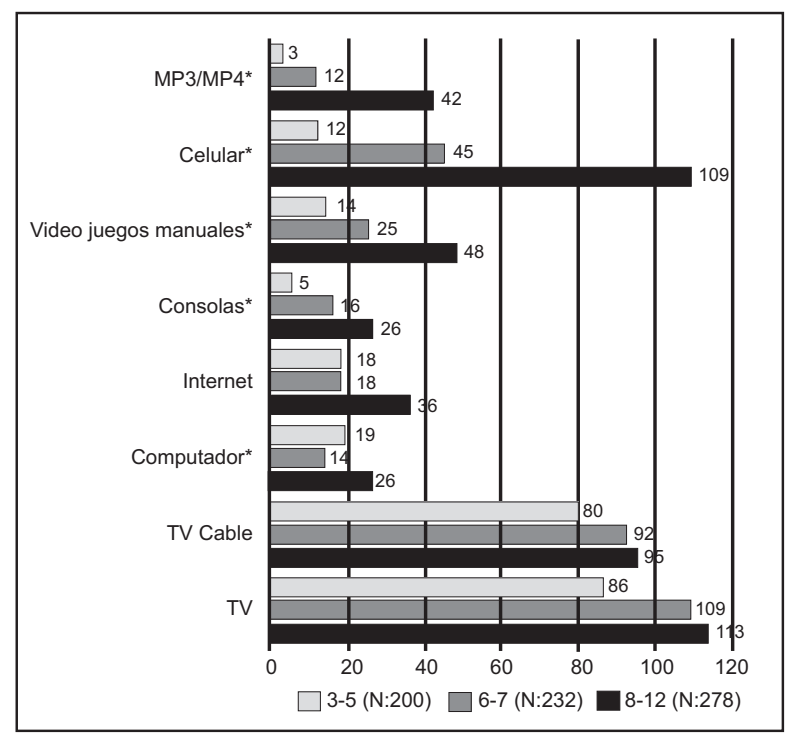

Figura 3. Disponibilidad de medios electrónicos según la edad de los niños $\left({ }^{*} p \leq 0,01\right)$ 
Cuadro 4. Factores asociados a la disponibilidad de medios electrónicos en la habitación del niño (variable de salida: mediana $\geq 1$ )

\begin{tabular}{|c|c|c|c|}
\hline Variable & RP & IC $95 \%$ & $\mathbf{P}$ \\
\hline $\begin{array}{l}\text { Madre trabaja fuera de } \\
\text { la casa (sí) }\end{array}$ & 1,16 & $1,00-1,35$ & 0,045 \\
\hline $\begin{array}{l}\text { Padre trabaja fuera de } \\
\text { la casa (sí) }\end{array}$ & 1,37 & $1,04-1,81$ & 0,023 \\
\hline $\begin{array}{l}\text { Edad del hijo (por año de } \\
\text { edad) }\end{array}$ & 1,08 & $1,04-1,12$ & $<0,0001$ \\
\hline Hermanos (sí) & 0,84 & $0,76-0,94$ & 0,003 \\
\hline
\end{tabular}

$\mathrm{N}=631$ observaciones; Linktest: 0,54

$38,7 \%\left(\mathrm{IC}_{95 \%}: 34,4-42,9\right)$ para ver más de dos horas de televisión entre semana y, de 58,8 \% $\left(\mathrm{IC}_{95 \%}\right.$ : 53,9 - 63,7), para el fin de semana. En el modelo multivariado, la edad de la mamá y la escolaridad del papá se asociaron en forma negativa con el uso de televisión por más de dos horas, tanto entre semana como los fines de semana, al igual que la manifestación de normas por parte de los padres. Por otro lado, la disponibilidad de televisor en el cuarto del niño se asoció positivamente, así como la concepción de los padres relacionada con las bondades de la televisión (cuadro 5).

Factores asociados a uso de consolas/videojuegos y computador más de una hora diaria. Se registró una prevalencia de $22,6 \%\left(\mathrm{IC}_{95 \%}: 18,1-27,0\right)$ para uso de computador y consolas más de una hora diaria entre semana y, de 43,9\% $\left(\mathrm{IC}_{95 \%}\right.$ : 39,4-48,5), para el fin de semana. En el modelo multivariado (cuadro 6), la actividad laboral fuera de la casa de la mamá, la disponibilidad de computador en el cuarto del hijo, el sexo masculino del hijo y la edad del mismo, aumentan en forma positiva y significativa la probabilidad de uso de computador y consolas por más de una hora diaria, entre semana y los fines de semana. Sólo la escolaridad de la mamá y vivir con los dos padres se asociaron en forma negativa.

\section{Discusión}

La importancia de este trabajo radica en que es el primero en Colombia en registrar la disponibilidad de medios electrónicos desde muy corta edad y en explorar los posibles factores asociados que contribuyan a entender esta dinámica en la sociedad actual. Los hallazgos son claros, no solo se dispone de medios y dispositivos electrónicos en el hogar, sino también, en la habitación de los niños. En particular, se dispone de televisor con acceso a transmisión por cable, computador e internet, así como consolas, videojuegos manuales y teléfonos móviles, lo cual se incrementa con la edad y es mayor en el sexo masculino (figuras 1

Cuadro 5. Factores asociados al uso de televisión por más de 2 horas diarias entre semana y los fines de semana

\begin{tabular}{|c|c|c|c|c|}
\hline Variable & $\begin{array}{c}\text { RP (IC } 95 \%) \\
\text { Entre semana * }\end{array}$ & $\mathbf{P}$ & $\begin{array}{c}\text { RP (IC } 95 \%) \\
\text { Fin de semana** }\end{array}$ & $\mathbf{P}$ \\
\hline Edad de la mamá (años) & $0,97(0,95-0,99)$ & 0,002 & ---- & \\
\hline Escolaridad del papá (años) & --- & & $0,98(0,96-1,0)$ & 0,03 \\
\hline \multicolumn{5}{|c|}{ Conceptos y normas sobre TV por los padres } \\
\hline TV buena para los niños (sí) & $1,28(1,04-1,58)$ & 0,018 & $1,24(1,08-1,42)$ & 0,002 \\
\hline TV límite 2 horas/día (sí) & $0,68(0,55-0,83)$ & $<0,0001$ & --- & \\
\hline TV control de programas (sí) & $0,65(0,50-0,84)$ & 0,001 & ---- & \\
\hline TV en el cuarto del niño (sí) & --- & & $1,14(1,0-1,30)$ & 0,06 \\
\hline
\end{tabular}

*N=647 observaciones; Linktest: 0,$91 ;{ }^{* *} \mathrm{~N}=620$ observaciones; Linktest: 0,40

Cuadro 6. Factores asociados a uso de consolas/videojuegos y computador por más de una hora al día

\begin{tabular}{lcccc}
\hline Variable & $\begin{array}{c}\text { RP (IC 95 \%) } \\
\text { Entre semana * }\end{array}$ & $\mathbf{P}$ & $\begin{array}{c}\text { RP (IC 95 \%) } \\
\text { Fin de semana** }\end{array}$ & $\begin{array}{l}\mathbf{P} \\
\text { Escolaridad de la mamá (años) }\end{array}$ \\
Trabaja la mamá & $0,95(0,92-0,99)$ & 0,02 & & \\
Computador en el cuarto & $1,84(1,04-3,25)$ & 0,037 & & \\
Sexo del hijo (masculino) & $2,04(1,22-3,40)$ & 0,006 & $1,55(1,15-2,05)$ & 0,004 \\
Edad del hijo (años) & $1,39(0,98-1,97)$ & 0,07 & $1,53(1,14-2,05)$ & 0,004 \\
Vive con los dos padres & --- & & $1,11(1,04-1,18)$ & 0,003 \\
\hline
\end{tabular}

${ }^{*} \mathrm{~N}=692$ observaciones; Linktest: 0,$15 ;{ }^{* *} \mathrm{~N}=699$ observaciones; Linktest: 0,68 
a 3), con cifras similares a las reportadas en otros estudios $(3,16)$.

En un estudio previo de población en Bucaramanga, de niños y jóvenes de 10 a 18 años, se registraron prevalencias, para ver televisión, de 95,3\% con un promedio semanal de 16,6 horas, para uso del computador, de 52,8\% con una media semanal de 6,7 horas, y para videojuegos, de $29,3 \%$ con un promedio de 4,7 horas semanales (17).

La Encuesta Nacional de la Situación Nutricional (3) mostró que el $62 \%$ de los niños y adolescentes ve televisión o juega videojuegos por dos o más horas diarias, registrándose un incremento de 4,5\% en la frecuencia de dos a cuatro horas al día en niños de 5 a 12 años, en comparación con los datos del año 2005. También, se registró que el tiempo excesivo de actividades de tipo sedentario aumenta con la edad y es más frecuente en adolescentes, en el área urbana y en los niveles de SISBEN más altos, aunque en el 2010 se registró una preocupación por el incremento de las mismas en el área rural y en el SISBEN 1.

Gómez, et al. (18), establecieron una asociación positiva entre los niveles de urbanización con ver o usar por dos o más horas diarias la televisión o los videojuegos, en niños de 5 a 12 años. Sin embargo, dadas las características del estudio, no se analizaron otras variables, como la disponibilidad de medios electrónicos adicionales y otros posibles factores asociados.

Las prevalencias de uso de televisión por más de dos horas diarias son inferiores a las de la Encuesta Nacional de la Situación Nutricional (3) y a las del estudio previo realizado en Bucaramanga (17). Sin embargo, cabe destacar la diferencia en el rango de edad estudiado (10 a 18 años), lo que seguramente afectó las prevalencias, pues ya se ha demostrado que la edad es un factor que se asocia positivamente con el uso de dispositivos electrónicos (4).

La diferencia de comportamientos entre semana y el fin de semana, refleja una dinámica cultural propia de nuestro contexto, en la cual el descanso de fin de semana se asocia con el predominio de actividades sedentarias en la población adulta, a la que se adhieren sus hijos por imitación o costumbre.

Hinkley, et al. (19), señalan que este campo de investigación apenas está emergiendo y establecen la necesidad de identificar los factores asociados a este tipo de comportamientos sedentarios, con el fin de orientar adecuadamente las estrategias de intervención. Con esta base, se analizan a continuación los factores asociados en este trabajo.

\section{Disponibilidad de medios electrónicos}

La escolaridad de la mamá y el ingreso de la mujer al mundo laboral por fuera de la casa $(64,5 \%)$, posiblemente contribuyen a incrementar los ingresos familiares, y son factores asociados con la disponibilidad de medios electrónicos en el hogar y en la habitación del hijo (cuadros 3 y 4); además, es posible que favorezcan un menor control del tiempo que los niños los usen, al estar al cuidado de otras personas mientras las madres trabajan.

Por otra parte, la adquisición de este tipo de productos para el hogar y los niños, también puede obedecer a una estrategia de compensación de los padres, en especial de la madre, por no estar disponibles para sus hijos; además, la percepción de inseguridad en las ciudades y la necesidad de los padres de disponer de tiempo para sí mismos y para las labores del hogar, generarían como respuesta la ocupación de los niños con este tipo de dispositivos.

En cuanto a la asociación negativa de contar con hermanos, se pueden plantear tres posibles explicaciones: la primera, que genera otras opciones como el juego, para que los niños ocupen su tiempo libre; la segunda, que hay menos ingresos disponibles para la adquisición de medios electrónicos; y la tercera, que existe la necesidad entre los hermanos de compartir los elementos de juego disponibles. Cabe señalar que estas posibles explicaciones deben ser evaluadas en otros estudios.

\section{Uso de medios electrónicos}

Se encontraron como factores asociados positivamente al uso de televisión por más de dos horas diarias y otros tipos de pantallas por más de una hora diaria, la concepción de que la televisión es buena para los niños, el trabajo de la mamá y la disponibilidad de computador en la habitación, sumado al género masculino y la edad de los hijos (cuadros 5 y 6). Estos hallazgos son similares y solo pueden compararse con los obtenidos en el trabajo de Vandewater, et al. (4), quienes evaluaron los factores asociados al incumplimiento de las recomendaciones de la American Academy of Pediatrics (7) relacionadas con ver televisión, es decir que no exceda las dos horas diarias en niños entre los 0 y 6 años; el autor encontró significativos la estructura familiar, categorizada como niños que viven con un solo padre $(\mathrm{OR}=2,27)$, el sexo femenino $(\mathrm{OR}=1,61)$, disponer de televisor en el 
cuarto del niño $(\mathrm{OR}=4,45)$, televisor en el hogar $(\mathrm{OR}=2,12)$ y la percepción de los padres relacionada con la ayuda que proporciona la televisión para el aprendizaje de los niños.

En este estudio, es importante señalar los factores asociados en forma negativa, entre los que están la escolaridad de los padres y vivir con ambos padres, lo que posiblemente se explica por un mejor acceso a la información y una mayor preocupación por las actividades de tiempo libre a las que se dedican sus hijos; esto también puede contribuir a la aplicación de normas y controles sobre el tiempo dedicado a ver televisión, así como a la calidad de los programas a los que tienen acceso sus hijos.

\section{Efectos potenciales del uso de medios electrónicos desde la primera infancia}

Nuestros hallazgos muestran que, a pesar de las diferencias socioculturales, la tecnología ya hace parte de la sociedad moderna colombiana, por lo cual es muy importante revisar brevemente sus efectos potenciales positivos o negativos, a corto, mediano y largo plazo, como posibles opciones para proyectos de investigación complementarios que contribuyan a la comprensión de esta dinámica de la población y a la propuesta de estrategias de intervención que puedan evaluarse posteriormente.

Las prevalencias encontradas para ver televisión entre semana $(96,1 \%$ ) y el fin de semana $(96,6 \%)$ son altas, y este uno de los factores más estudiados que contribuye al sobrepeso y la obesidad desde la edad preescolar; sin embargo, aún no se sabe mediante qué mecanismo se da esta asociación. Se ha postulado que puede explicarse por la ingestión de comidas no saludables hipercalóricas mientras se ve televisión y, además, se ha planteado que los contenidos de la televisión pueden tener un efecto negativo sobre las niñas, al inducir problemas de anorexia y bulimia, en especial cuando están llegando a la pubertad (20-24).

Los efectos de la exposición a ver televisión más de dos horas diarias sobre las destrezas sociales y el comportamiento de los niños a la edad de 5,5 años, fueron analizados por Mistry, et al. (25), quienes encontraron que la exposición sostenida entre los 30 y 60 meses de edad se asoció con problemas de sueño y de atención, así como con comportamientos agresivos y falta de interacción social.

Las prevalencias de disponibilidad de celulares también fueron altas $y$, además, se incrementaron con la edad (figuras 2 y 3 ). Sin embargo, los efectos son controversiales. Se ha registrado que un uso mayor de cinco minutos en horas de la mañana aumenta los problemas de irritabilidad $(\mathrm{OR}=1,64)$, dolores de cabeza $(O R=1,55)$ y fatiga $(O R=1,76)$ en horas del medio día. En los niños entre los 8 y 10 años, se encontraron problemas de concentración en horas de la noche $(\mathrm{OR}=1,55)$, por el uso de celulares en horas de la tarde (26).

Por otra parte, reconociendo que es imposible detener la dinámica mundial relacionada con el acceso y uso de este tipo de dispositivos, en la actualidad se plantea la necesidad de utilizar los medios en forma positiva, para incrementar los hábitos alimenticios saludables y las conductas pro-sociales, y para disminuir el consumo de alcohol, el de tabaco y la iniciación temprana de relaciones sexuales (24). Además, se propone la necesidad de educar a los padres para controlar no solo el tiempo, sino el contenido y la calidad de los programas de televisión y de las redes de información a las que acceden sus hijos, así como de implementar normas en los hogares para su utilización, siguiendo las recomendaciones de las organizaciones internacionales (7-9).

Es importante señalar las limitaciones de este trabajo, las cuales pueden ser objeto de estudio en otros proyectos. La primera se relaciona con la naturaleza del diseño, que por ser de corte transversal no permite establecer causalidad, solo factores asociados $(27,28)$. En segundo lugar existe un potencial sesgo de selección por falta de respuesta, considerando el porcentaje de personas que respondieron la encuesta $(59,2 \%)$ del total de encuestas remitidas, y un sesgo potencial de clasificación por la calidad de respuestas obtenidas a partir del recuerdo de las actividades de los niños, registradas por sus padres, lo que podría sobreestimar o subestimar las asociaciones detectadas. Esta apreciación se basa en la moderada reproducibilidad de las respuestas emitidas en la prueba piloto que se hizo. Por otra parte, no se indagó por la calidad y el contenido de la televisión que ven los niños, debido a que esta variable no se consideró dentro del objetivo de este estudio; asimismo, se desconoce cómo pueden interactuar factores contextuales de orden familiar y de relaciones interpersonales que modifiquen la dinámica aquí estudiada.

A pesar de las limitaciones señaladas, los hallazgos de este trabajo son relevantes por varias razones. Una de ellas es que corresponden a 
una muestra de base de población seleccionada aleatoriamente, que refleja el fenómeno social bajo estudio con un nivel de precisión que permite extrapolar los hallazgos a la población de los municipios seleccionados. Este es el primer estudio en Colombia dirigido a llamar la atención sobre una dinámica social actual muy preocupante, pero aún desconocida en nuestro contexto, y proporciona cifras relevantes para proponer nuevas propuestas, así como la necesidad de divulgar las recomendaciones internacionales para su uso adecuado en niños desde la primera infancia.

Esta preocupación se basa también en el desarrollo permanente de nuevos y llamativos dispositivos electrónicos, los cuales son promocionados con campañas publicitarias muy fuertes, que inducen a la sociedad de consumo a utilizarlos en forma permanente y masiva. Este comportamiento no solo hace parte de la vida cotidiana en adultos; cada vez niños más jóvenes se ven afectados por la necesidad inducida de disponer de los mismos, en especial los de última generación, lo que muestra una dinámica social bastante compleja, que puede tener impacto en indicadores como dificultad de adaptación al medio social, baja capacidad para resolver conflictos, comportamientos agresivos, bajo rendimiento académico y, además, escasa interacción de los niños y jóvenes con sus pares, padres y tutores, entre otros aspectos de los que no se tiene información en nuestro contexto. Sin embargo, pueden convertirse también en un recurso educativo y de motivación para la práctica regular de actividad física, así como favorecer la interacción social, siempre que sus contenidos y abordajes obedezcan a objetivos claros y puedan ser evaluados.

Es importante considerar también que hay una marcada diferencia entre las generaciones actuales de niños en su primera infancia, comparados con los niños de generaciones precedentes, pues la tecnología hace parte de su vida desde el nacimiento, hecho que debe aprender a manejarse, tanto por los padres como por las instituciones educativas, con el fin de utilizarlos positivamente para contribuir a la educación de las nuevas generaciones y disminuir los potenciales efectos negativos que a la fecha han sido planteados.

\section{Agradecimientos}

Los autores agradecen la participación de los representantes legales de las instituciones educativas participantes, así como de los padres de familia, quienes diligenciaron la encuesta y permitieron recolectar la información que aquí se presenta. Igualmente, agradecen el apoyo financiero brindado por la Universidad Industrial de Santander para la ejecución del mismo.

\section{Conflicto de intereses}

Los autores manifiestan no registrar conflicto de interés alguno durante la ejecución y desarrollo del trabajo que aquí se presenta.

\section{Financiación}

Este trabajo contó con la financiación de la Vicerrectoría de Investigación y Extensión de la Universidad Industrial de Santander y fue registrado con el código 5668 en la Dirección de Investigación de la Facultad de Salud de la Universidad Industrial de Santander.

\section{Referencias}

1. Biddle SH, Gorety T, Marshall SJ, Mardey I, Cameron N. Physical activity and sedentary behaviors in youth: Issues and controversies. J R Soc Promot Health. 2004;124: 2933. http://dx.doi.org/10.1177/146642400312400110

2. Organización Mundial de la Salud. Estrategia mundial sobre régimen alimentario, actividad física y salud. Fecha de consulta: 10 de marzo de 2011. Disponible en: http:// www.who.int/dietphysicalactivity/childhood/es/index.html.

3. Instituto Colombiano de Bienestar Familiar. Encuesta Nacional de la Situación Nutricional en Colombia 2005. [Fecha de consulta: 24 de enero de 2010. Disponible en: http://nutrinet,org/index,php?option=com_remository\&ltemi $d=110$ \&func=fileinfo\&id=385.

4. Vandewater EA, Rideout VJ, Wartella EA, Huang X, Lee JH, Shim M. Digital childhood: Electronic media and technology use among infants, toddlers and preschoolers. Pediatrics. 2007;119:e1006-15. http://dx.doi.org/10.1542/ peds.2006-1804

5. Levy PS, Lemeshow S. Sampling of populations: Methods and applications. New York: John Wiley \& Sons; 1994.

6. Spurrier NJ, Magarey AA, Golley R, Curnow F, Sawyer MG. Relationships between the home environment and physical activity and dietary patterns of preschool children: $A$ cross-sectional study. Int J Behav Nutr Phys Act. 2008;5:31. http://dx.doi.org/10,1186/1479-5868-5-31

7. Davis MM, Gance-Cleveland B, Hassink S, Johnson R, Paradis G, Resnicow K. Recommendations for prevention of childhood obesity. Pediatrics. 2007;120(Suppl.4):S22953. http://dx.doi.org/10.1542/peds.2007-2329E

8. National Association for Sport and Physical Education. Active start: A statement of physical activity guidelines for children from birth to five years. Reston, VA: NASPE Publications; 2002.

9. Australian Government. Department of Health and Aging (DoHA). Get up and grow: Healthy eating and physical activity for early childhood. Fecha de consulta 25 de septiembre de 2012. Disponible en: http://www.health. gov.au/internet/main/publishing.nsf/Content/EDFEDB5884 60BCE3CA25762B00232A13/\$File/gug-directorscoord.pdf . 
10. Pagano M. Principles of biostatistics. Belmont: Duxbury Press; 1993.

11. Siegel S, Castellan NJ. Nonparametric statistics for the behavioral sciences. Second edition. Singapore: McGraw Hill; 1988.

12. Wacholder S. Binomial regression in GLIM: Estimating risk and risk differences. Am J Epidemiol. 1986;123:174-84.

13. Zochetti C, Consonni D, Vertais PA. Relationship between prevalence rate ratios and odds ratios in cross-sectional studies. Int J Epidemiol. 1997;26:220-3. http://dx.doi. org/10.1093/ije/26.1.220

14. Greenland S. Modeling and variable selection in epidemiologic analysis. Am J Public Health. 1999;79:340-9.

15. StataCorp. Stata: Release 12. Statistical software. College Station, TX: StataCorp LP; 2011.

16. Certain LK, Kahn RS. Prevalence, correlates, and trajectory of television viewing among infants and toddlers. Pediatrics. 2002;109:634-42. http://dx.doi.org/10.1542/peds.109.4.634

17. Camargo DM, Orozco LC, Herrera E. Dolor músculo esquelético en adolescentes. Prevalencia y factores asociados. Salud UIS. 2007;39:165-75.

18. Gómez LF, Lucumí DI, Parra DC, Lobelo F. Niveles de urbanización, uso de televisión y video-juegos en niños colombianos: posibles implicaciones en salud pública. Rev Salud Pública. 2008;10:505-16. http://dx.doi.org/10.1590/ S0124-00642008000400001.

19. Hinkley T, Salmon J, Dókely A, Trost SG. Correlates of sedentary behaviours in preschool children: A review. Int J Behav Nutr Phys Act. 2010;7:66. http://dx.doi. org/10.1186/1479-5868-7-66

20. Raynor HA, Jelalian E, Vivier PM, Hart CN, Wing RR. Parent-reported eating and leisure time activity selection patterns related to energy balance in preschool- and schoolaged children. J Nutr Educ Behav. 2009;41:19-26. http:// dx.doi.org/10.1016/j.jneb.2008.03.008
21. Bryant MJ, Lucove JC, Evenson KR, Marshall S. Measurement of television viewing in children and adolescents: A systematic review. Obes Rev. 2007;8:197209. http://dx.doi.org/10.1111/j.1467-789X.2006.00295.x

22. Marshall SJ, Biddle SJH, Gorely T, Cameron N, Murdey I. Relationships between media use, body fatness and physical activity in children and youth: A meta-analysis. Int J Obes. 2004;28:1238-46. http://dx.doi.org/10.1038/ sj.ijo,0802706.

23. Hesketh K, Wake M, Graham M, Waters E. Stability of television viewing and electronic game/computer use in a prospective cohort study of Australian children: Relationship with body mass index. Int J Behav Nutr Phys Act. 2007;4:60. http://dx.doi.org/10.1186/1479-5868-4-60

24. Ray M, Ramjat K. Effect of electronic media on children. Ind Pediatr. 2010;47:561-8.

25. Mistry KB, Minkovitz CS, Strobino DM, Borzekowski DL. Children's television exposure and behavioural and social outcomes at 5.5 years: Does timing of exposure matters? Pediatrics. 2007;120:762-9. http://dx.doi.org/10.1542/peds. 2006-3573

26. Heinrihc S, Thomas S, Heumann C, von Kries R, Radon K. Association between exposure to radiofrequency electromagnetic fields assessed by dosimetry and acute symptoms in children and adolescents: A population based cross-sectional study. Environ Health. 2010;9:75. http:// dx.doi.org/10.1186/1476-069X-9-75

27. Kraemer CH, Kazdin AE, Offord DR, Kessler RC, Jensen PS, Kupfer DJ. Coming to terms with the terms of risk. Arch Gen Psychiatry. 1997;54:337-43. http://dx.doi.org/10.1001/ archpsyc.1997.01830160065009.

28. Kraemer CH, Stice E, Kazdin A, Offord D, Kupfer D. How do risk factors work together? Mediators, moderators, and independent, overlapping, and proxy risk factors. Am J Psychiatry. 2001;158:848-56. 